Z Rheumatol 2020 · 79:826-827 https://doi.org/10.1007/s00393-020-00892-2 Online publiziert: 21 . September 2020 (c) Springer Medizin Verlag GmbH, ein Teil von Springer Nature 2020

\author{
J. Braun ${ }^{1,2}$ für den Vorstand der DGRh • B. Bühring ${ }^{1,2}$ für den Vorstand der DGRh \\ 'Rheumazentrum Ruhrgebiet, Herne, Deutschland \\ ${ }^{2}$ Ruhr Universität Bochum, Bochum, Deutschland
}

\title{
Nachruf der Deutschen Gesellschaft für Rheumatologie auf Prof. Dieter Felsenberg
}

tektur und Knochenfestigkeit. Wissenschaftliche Schwerpunkte waren neben der Osteoporose der Muskel- und Knochenstoffwechsel, die Biomechanik des Knochens, seltene Knochenerkrankungen, Sportmedizin, die Arthrose, die rheumatoide Arthritis und die $\mu$-CTund die pQCT-Technologie sowie der Muskel- und Knochenstoffwechsel in der Schwerelosigkeit. Bei der Durchführung vieler verschiedenen klinischer, pharmakologischer und - nicht-pharmakologischer Studien war er an verantwortlicher Stelle involviert, u.a. im Bereich der Prävention und Therapie der postmenopausalen Osteoporose, der Osteoporose des Mannes, aber auch der Entwicklung von multimodalen Therapiekonzepten, der Behandlung von Gonarthrose, etwa mittels des GalileoVibrationstrainings und der Differenzialdiagnose von ,Early Onset Osteoporosis. $\mathrm{Zu}$ den Themen Osteoporose, MuskelKnochen-Interaktion und Biomechanik des Knochens hat er insgesamt über 330 Veröffentlichungen realisiert und viele Promotionen betreut. Über viele Jahre war er gemeinsam mit Vertretern der DGRh in der Leitlinienkommission des Dachverbandes Osteologie (DVO) aktiv.

Dieter Felsenberg war ein Querdenker, der mit seiner Forschung etablierte Konzepte hinterfragte und so die Wissenschaft $\mathrm{zu}$ neuen Erkenntnissen brachte. Er war unkonventionell und bodenständig - sowohl seine Holzclogs als auch seine herzlichen Umarmungen waren auf nationalen und internationalen Kongressen bekannt und er wurde für sein Wissen und seine gewinnende Art weltweit respektiert. Als streitbarer
Wissenschaftler prägte er die Forschung zur Muskel- und Knocheninteraktion, deren Ideen sich in einem Jahreskongress „Muskeln und Knochen - neue Welten!“ widerspiegelten. Forschungsprojekte zu Muskel- und Knocheninteraktion bei Immobilisation sowie unter Schwerelosigkeit bildeten einen Schwerpunkt in Kooperation mit internationalen Weltraumbehörden und sportmedizinischen Zentren. In der klinischen Forschung trug er wesentlich dazu bei, das Krankheitsbild der Osteoporose in Prävention, Behandlung und Epidemiologie besser $\mathrm{zu}$ verstehen.

Parallel zur Forschung betreute Prof. Felsenberg viele Patienten in seiner osteologischen Schwerpunktsprechstunde, womit er in gewisser Weise und translationale Medizin lebte - von der Basisforschung bis zur klinischen Anwendung. Neben seinen wissenschaftlichen Anstrengungen und Erfolgen blieb er dabei aber vor allen Dingen immer auch ein gewissenhafter und einfühlsamer Arzt. Seit 10/2018 stand er noch bis vor einigen Monaten im Rahmen einer Spezialsprechstunde für seltene Muskelund Knochenkrankheiten in einer großen Rheumapraxis in Berlin für seine Patienten zur Verfügung.

Da ihm die Ausbildung junger Kollegen und Nachwuchswissenschaftler sehr am Herzen lag, war Prof. Felsenberg Mitbegründer der Deutschen Akademie für Osteologische und Rheumatologische Wissenschaften (DAdorW). Er war Mitglied in zahlreichen nationalen und internationalen Fachgesellschaften. Zudem war er Mitgründer des Zentrums für Weltraummedizin an der FU Berlin, 


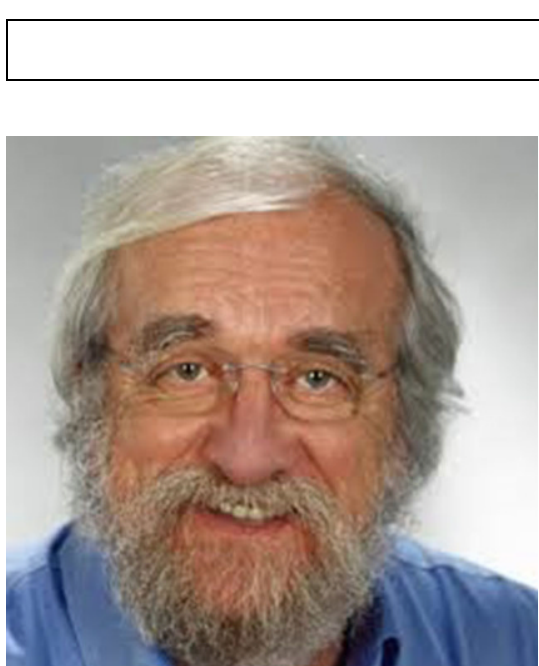

Abb. 1 ॥ Prof. Dieter Felsenberg

der Deutschen Gesellschaft für Muskel- und Knochenforschung (DGfMK) sowie der International Society of Musculoskeletal and Neuronal Interaction (ISMNI). Im Jahr 2004 implementierte er das Deutsche Kiefernekrose Register. Im Jahr 2008 war er Mitbegründer der Osteologie-Akademie OSTAK, einer Tochtergesellschaft des DVO. Seit April 2011 lehrte er als Honorarprofessor an der School of Science, Technology and Health an der University of Suffolk, UK.

Dieter Felsenberg liebte seine Arbeit und begeisterte Kollegen, Mitarbeiter, Nachwuchswissenschaftler und Patienten mit seinem ansteckenden Enthusiasmus und seiner Lebensfreude. Die Zusammenarbeit mit ihm war geprägt von Herzlichkeit und Humor, gelebter Gemeinschaft, Großzügigkeit und Offenheit, verbunden mit Integrität und hohem wissenschaftlichen Anspruch. Beim DGRh-Kongress 2018 sprach er im Rahmen des Osteoporose/ Sarkopenie Symposiums über die neuesten Forschungsergebnisse in der ZytokinKommunikation zwischen Muskeln und anderen Organen, z. B. mit Hilfe von Myokinen.

Mit ihm haben wir einen national und international herausragenden Wissenschaftler, engagierten Arzt, unterstützenden Mentor und wichtigen Freund verloren. Das Thema der Knochen- und Muskeleinheit hat durch seinen Enthusiasmus Einzug in das Gebiet der Knochenforschung erhalten. Wir werden uns an ihn immer als einen diskussionsfreudigen, innovativen, originellen aber auch lebenslustigen Menschen erinnern, der für sein Thema begeistern konnte.

Viele Rheumatolog*innen kannten Prof. Felsenberg und schätzten seine interessanten Vorträge. Die DGRh schließt sich der langen Liste von Trauernden an, schätzt ihn als Freund der Rheumatologie und wird sein Andenken zur Ermutigung für engagierte wissenschaftliche Tätigkeit für zukünftige Generationen bewahren.

Für den Vorstand der DGRh Jürgen Braun und Björn Bühring

\section{Ausgewählte Publikationen mit Dieter Felsenberg}

- Buehring B, ..., Felsenberg D ... Changes in lower extremity muscle function after 56 days of bed rest. J Appl Physiol 2011;111(1):87-94.

- Karberg K, ..., Felsenberg D, Braun J. Bone loss is detected more frequently in patients with ankylosing spondylitis with syndesmophytes. J Rheumatol 2005; 32(7):1290-1298.

\section{Korrespondenzadresse}

\section{Prof. Dr. J. Braun}

Rheumazentrum Ruhrgebiet Claudiusstr. 45, 44649 Herne, Deutschland juergen.braun@elisabethgruppe.de

\section{Verschobene Operationen im Corona-Shutdown}

Kataraktoperationen, Kniegelenkimplantationen und Entfernung der Rachenmandeln sind die am häufigsten verschobenen Operationen während des Corona-Shutdowns.

Die Auswertung von bundesweiten Daten hat einen Rückgang der Operationen während des Shutdowns im April von 41 Prozent ergeben. Am häufigsten unter den verschobenen Eingriffen waren Kataraktoperationen mit -79 Prozent, die Entfernung der Rachenmandeln mit -82 Prozent und die Implantation von Kniegelenkendoprothesen mit -80 Prozent. Etwas häufiger unter den verschobenen Operationen wurden noch Kniegelenkathroskopien, -67 Prozent, durchgeführt sowie die Entfernung von Osteosynthesematerial, -51 Prozent. Demgegenüber hat die Anzahl der Kaiserschnitte, der versorgten Knochenbrüche und Blinddarmentzündungen gar nicht beziehungsweise nur sehr geringfügig abgenommen.

„Es geht jetzt darum, Lehren aus dieser Zeit für eine etwaige zweite Welle zu ziehen. Dazu gehört auch, die Pandemiepläne so umzuarbeiten, dass bei einem Anstieg des Infektionsgeschehens, die Krankenhäuser ihren Betrieb mit einem Anteil elektiver Operationen weiter aufrechterhalten können." kommentiert Prof. Dr. Dr. Hans-Joachim Meyer, Präsident des Berufsverbands der Deutschen Chirurgen die Ergebnisse.

Das Ziel des medizinischen Shutdowns wurde erreicht. Auswirkungen der Verschiebungen müssen genau untersucht und Pandemiepläne künftig regional und lokal angepasst werden.

\section{Quelle: Berufsverband der Deutschen Chirurgen e.v. (BDC), www.bdc.de}

